\title{
Verkehrliche Wirkung autonomer Fahrzeuge
}

\author{
Bernhard Friedrich
}

\section{Inhaltsverzeichnis}

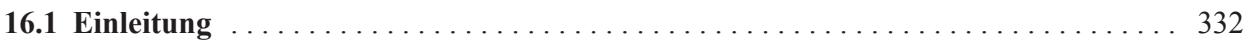

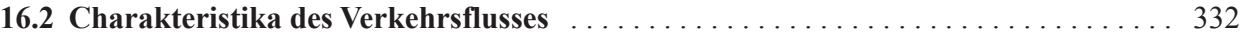

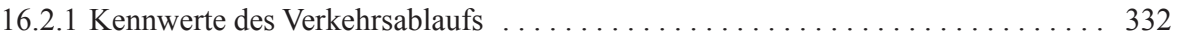

16.2.2 Verkehrsflusstheorie ............................. 333

16.2.3 Modell für stationäre Verkehrszustände - Fundamentaldiagramm . . . . . . . . 335

16.2.4 Kapazität und Stabilität . . . . . . . . . . . . . . . . . . . . . . . . . . 336

16.3 Verkehrliche Wirkung autonomer Fahrzeuge $\ldots \ldots \ldots$. . . . . . . . . . . . . . . . 339

16.3.1 Streckenabschnitte von Autobahnen . . . . . . . . . . . . . . . . . . . . . . 339

16.3.2 Knotenpunkte mit Lichtsignalanlage $\ldots \ldots \ldots \ldots \ldots \ldots \ldots \ldots \ldots \ldots \ldots . \ldots \ldots 4$

16.3.3 Abschätzung der Effizienzgewinne durch autonomes Fahren . . . . . . . . . . 346

16.4 Zusammenfassung und Ausblick $\ldots \ldots \ldots \ldots \ldots \ldots \ldots \ldots \ldots \ldots \ldots \ldots \ldots \ldots \ldots \ldots$

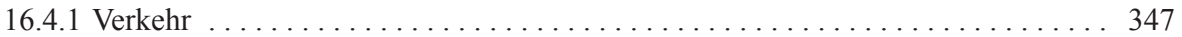

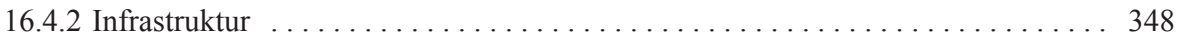

16.4.3 Kooperation .................................... 349

Literatur . . . . . . . . . . . . . . . . . . . . . . . . . . . . 349

B. Friedrich $(\bowtie)$

Technische Universität Braunschweig, Institut für Verkehr und Stadtbauwesen, Deutschland friedrich@tu-braunschweig.de 


\subsection{Einleitung}

Autonome Fahrzeuge nehmen selbstständig am Verkehr teil, ohne dass sie den Menschen als Überwacher oder Entscheider benötigen. Ihren Fahrgästen bieten autonome Fahrzeuge einen Komfortgewinn, da keine Fahraufgaben geleistet werden müssen. Einem Personenkreis, der bislang aufgrund von Mobilitätseinschränkungen von der Teilhabe am öffentlichen Leben teilweise oder ganz ausgeschlossen ist, bieten autonome Fahrzeuge neue Chancen für dessen Mobilität.

Neben den Vorteilen, die autonome Fahrzeuge für deren Nutzer bieten mögen, ist der gesellschaftliche Nutzen, der mit ihrer Verbreitung verbunden ist, von Interesse. Denn es ist offensichtlich, dass autonomes Fahren zu keinen Einbußen bei der Sicherheit oder der Effizienz des Straßenverkehrs führen darf, sondern diese verbessern soll. Dieser Beitrag untersucht in diesem Zusammenhang die verkehrlichen Wirkungen autonomer Fahrzeuge hinsichtlich der Effizienz, mit der die bestehende Infrastruktur genutzt werden könnte.

Die Effizienz von Verkehrsanlagen wird durch ihre Kapazität bestimmt. Auf den Fernstraßen ist die Kapazität im Wesentlichen durch den maximal möglichen Verkehrsfluss auf den Streckenabschnitten sowie die maximal möglichen Verflechtungs- und Ein- bzw. Ausfädelungsverkehrsstärken an den planfreien Knotenpunkten bestimmt. Im Stadtstraßennetz und auf Landstraßen mit Ortsdurchfahrten sind die Kapazitäten an den Knotenpunkten maßgebend und somit meist von den Lichtsignalanlagen abhängig. Während die Kapazität an Lichtsignalanlagen von den Zeitbedarfswerten bestimmt wird, die die einzelnen Fahrzeuge benötigen, um in der anfahrenden Kolonne den Knotenpunkt zu passieren, werden die Kapazitäten von Autobahnabschnitten durch die Instabilität bestimmt, die sich bei hohen Verkehrsstärken einstellt und zum Stau führt.

Für das Verständnis der Überlegungen zu den verkehrlichen Wirkungen autonomer Fahrzeuge werden in diesem Beitrag zunächst die Kennwerte des Verkehrsablaufs und ihre Zusammenhänge erläutert. Auf diesen Grundlagen aufbauend werden dann Überlegungen zum Einfluss autonomer Fahrzeuge auf die Kapazitäten von freien Strecken im Zuge von Fernstraßen sowie von Knotenpunkten mit Lichtsignalanlagen abgeleitet. Die Auswirkungen autonomer Fahrzeuge auf die Verbindungsqualitäten von Fahrten, die über unterschiedliche Infrastrukturelemente verlaufen, können mit diesen Überlegungen nicht ausreichend beschrieben werden. Dennoch liefern die Betrachtungen eine erste Einschätzung, welche Optimierungspotenziale für die Effizienz des Verkehrsablaufs mit autonomen Fahrzeugen verbunden sein könnten.

\subsection{Charakteristika des Verkehrsflusses}

\subsubsection{Kennwerte des Verkehrsablaufs}

Zur mathematischen Abbildung des Verkehrsablaufs wird von einer Abstraktion des Wegenetzes, der Fahrzeuge, der Fahrer sowie deren Verhalten, d. h. von vereinfachenden Annahmen ausgegangen. 
Das Wegenetz wird z. B. in freie Strecken und Knotenpunkte aufgeteilt. Gegenstand der Untersuchungen ist dann entweder die freie Strecke oder der Knotenpunkt, wobei gleichbleibende Bedingungen wie Ebenheit, ausreichende Sicht, trockene Fahrbahn etc. vorausgesetzt werden. Hinsichtlich Fahrer und Fahrzeug wird u. a. angenommen, dass deren Eigenschaften wie Reaktionszeit, Risikobereitschaft bzw. technischer Zustand empirisch nachgewiesenen Verteilungen folgen.

Es wird zwischen mehreren Formen der Beschreibung des Verkehrsablaufs unterschieden. Die mikroskopische Abbildungsweise beschreibt die verkehrlich relevanten Merkmale eines Einzelfahrzeugs $i$ :
- zeitlicher Abstand
$t_{i}(s)$
- räumlicher Abstand
$x_{i}(m)$,
- Geschwindigkeit
$v_{i}(\mathrm{~km} / \mathrm{h})$.

Die makroskopische Abbildungsweise betrachtet eine Menge von Fahrzeugen und die relevanten Kennwerte eines Verkehrsstroms:

- Verkehrsstärke

$$
\begin{array}{ll}
\text { - Verkehrsstärke } & q(F z / h), \\
\text { - Verkehrsdichte } & k(F z / k m),
\end{array}
$$$$
\text { - mittlere Geschwindigkeit } v(\mathrm{~km} / \mathrm{h}) \text {. }
$$

Der Verkehrsablauf lässt sich erfassen durch Messungen der Kennwerte an einem bestimmten Querschnitt über ein Zeitintervall $d t$ durch sogenannte lokale Beobachtungen oder Messungen zu einem bestimmten Zeitpunkt über ein Wegintervall $d x$ als sogenannte momentane Beobachtungen (s. Abb. 16.1).

\subsubsection{Verkehrsflusstheorie}

Weder die makroskopischen Kennwerte $v, q$ und $k$ noch die entsprechenden mikroskopischen Werte definieren für sich allein einen Verkehrszustand. Dazu ist die Kenntnis ihrer gegenseitigen Abhängigkeiten Voraussetzung. Die drei makroskopischen Größen Verkehrsstärke, Verkehrsdichte und momentane (streckenbezogene) Geschwindigkeit sind durch die Gleichung

$$
q=k \cdot v(k)
$$

miteinander verknüpft. Messungen der Verkehrsstärke und der mittleren Geschwindigkeit ergaben eine nachweisbare Abnahme der Geschwindigkeit bei zunehmender Verkehrsstärke, d. h. mit zunehmender gegenseitiger Beeinflussung der Fahrzeuge.

Eines der ersten Modelle zur Beschreibung des Verkehrsablaufs auf der freien Strecke resultiert aus Beobachtungen von Greenshields [5], der den Zusammenhang zwischen 


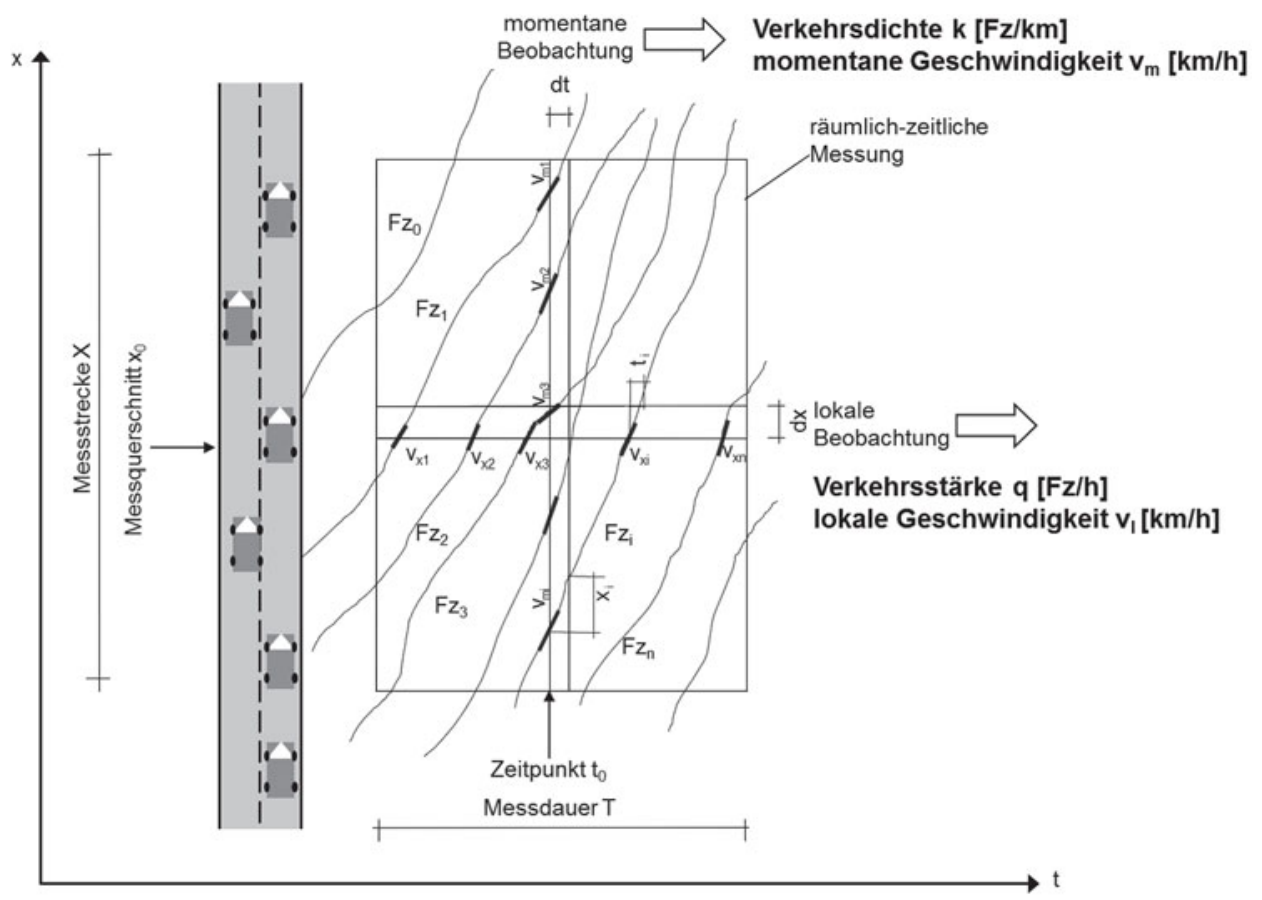

Abb. 16.1 Systematik lokaler und momentaner Messungen. Unterschiedliche Geschwindigkeiten ergeben sich für die lokal bzw. momentan erfassten Einzelgeschwindigkeiten

Geschwindigkeit $v$ und der Verkehrsdichte $k$ erforschte. Mithilfe der Regressionsrechnung stellte er einen linearen Zusammenhang für $v=v(k)$ fest

$$
v(k)=v_{f}-v_{f} / k_{\max } \cdot k=v_{f} \cdot\left(1-k / k_{\max }\right)
$$

wobei $v_{f}$ die freie Geschwindigkeit und $k_{\max }$ die maximale Verkehrsdichte darstellen.

Eingesetzt in die Gleichung $q=v \cdot k$ ergibt sich damit ein parabolischer Zusammenhang zwischen Verkehrsstärke und Verkehrsdichte in der Form:

$$
q(k)=v_{f} \cdot\left(k-k^{2} / k_{\max }\right)
$$

Gleichungen mit diesen Kenngrößen werden als Zustandsgleichungen und ihre grafische Repräsentation als Fundamentaldiagramme des Verkehrs bezeichnet. 


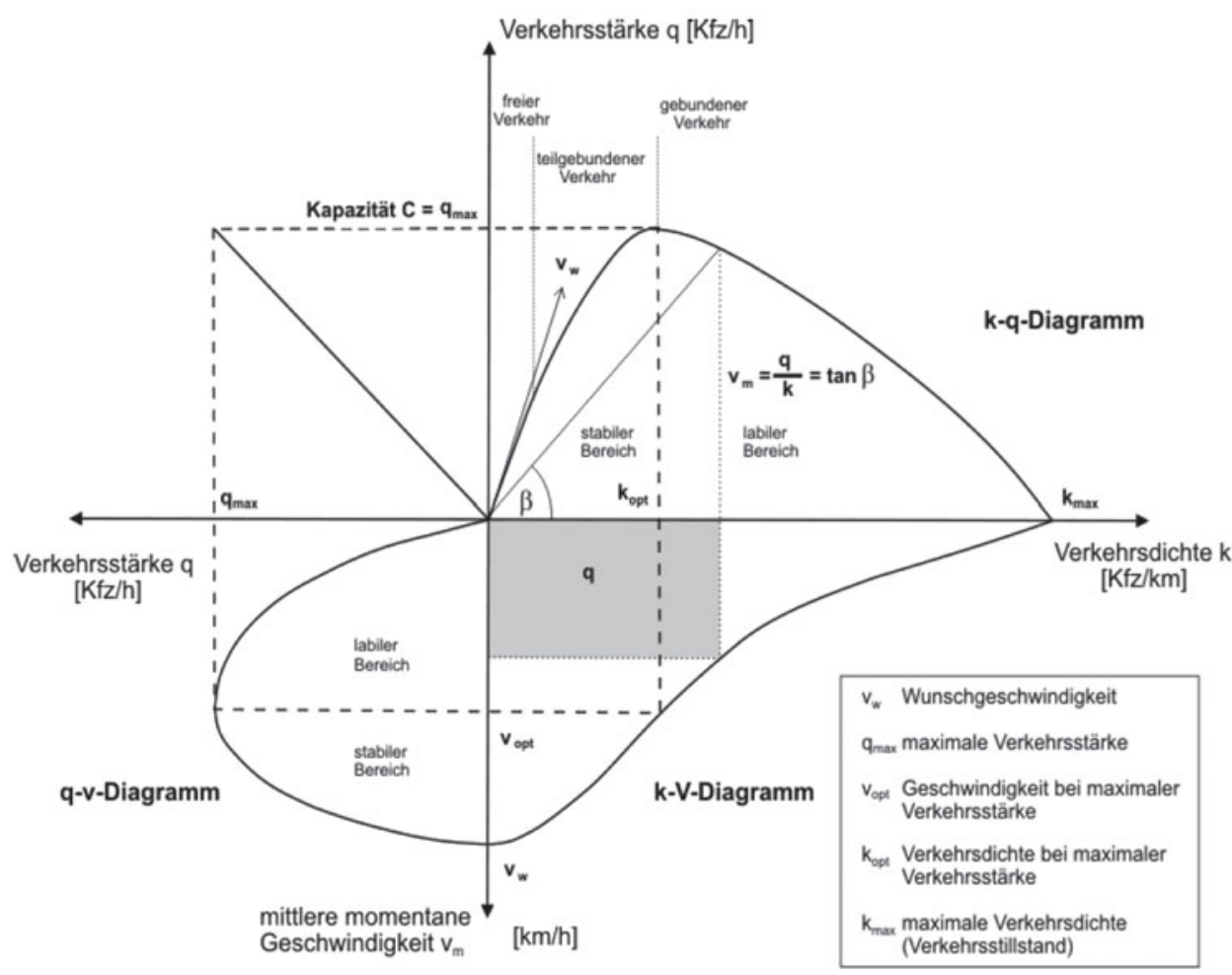

Abb. 16.2 Ansichten des Fundamentaldiagramms [3]

\subsubsection{Modell für stationäre Verkehrszustände - Fundamentaldiagramm}

Das Fundamentaldiagramm ist die grafische Abbildung der Zustandsgleichung des Verkehrs, also des funktionalen Zusammenhangs zwischen den Kenngrößen Verkehrsstärke $q$, Verkehrsdichte $k$ und mittlerer momentaner, d.h. abschnittsbezogener Geschwindigkeit $v$ und stellt eine Kurve im dreidimensionalen Raum dar. Die orthogonalen Projektionen der Kurve auf die von jeweils zwei Kenngrößen aufgespannten Ebenen ergeben die in Abb. 16.2 gezeigten bekannten Ansichten des Fundamentaldiagramms. Die resultierenden drei Diagramme ermöglichen vielfältige Aussagen über die Charakteristik des Verkehrsflusses an einem Querschnitt und werden als $q$ - $v$-Diagramm, $q$ - $k$-Diagramm und $k$ - $v$-Diagramm bezeichnet.

Das Fundamentaldiagramm lässt erkennen, dass bei gleicher Verkehrsstärke $q_{l}$ zwei unterschiedliche Qualitäten des Verkehrsablaufs auftreten können. Die Schwelle $q_{\max }$ trennt für $q_{i}<q_{\max }$ den Bereich hoher Geschwindigkeiten bei geringen Verkehrsdichten, also den freien und stabilen Verkehrsablauf, vom Bereich mit relativ niedrigen Geschwindigkeiten und hohen Verkehrsdichten, dem Bereich des instabilen und gestörten Verkehrsablaufs. Empirische Untersuchungen zeigen, dass der Übergang zwischen den beiden Bereichen 
Abb. 16.3 Fundamentaldiagramm mit getrennten Bereichen für stabilen und instabilen Verkehr für einzelne Fahrstreifen bzw. zweistreifige Fahrbahn [8]

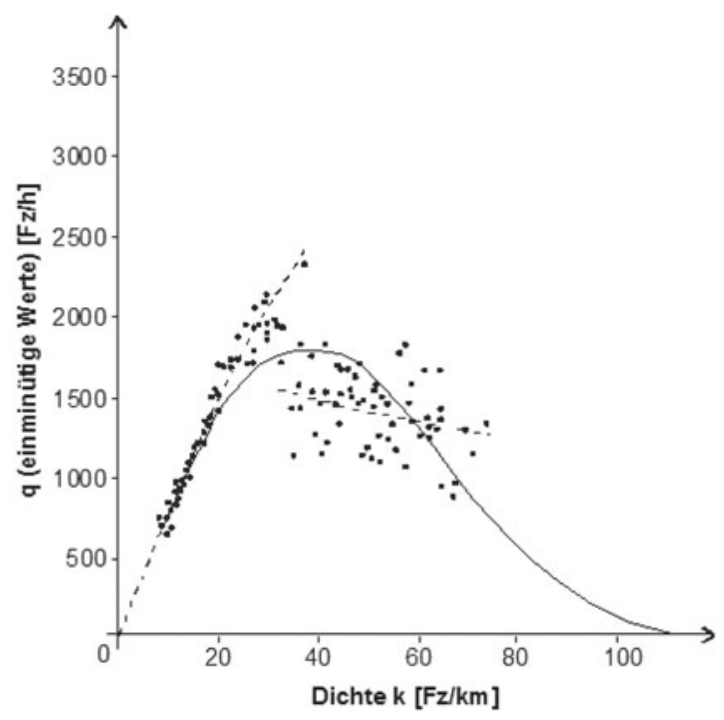

nicht wie in der idealisierten Form in Abb. 16.2 kontinuierlich verläuft. Vielmehr erfolgt bei hohen Verkehrsstärken, ausgelöst durch Störungen im Verkehrsablauf, ein Übergang aus dem stabilen in den instabilen Bereich, der mit einem deutlichen Rückgang der Verkehrsstärke verbunden ist (s. Abb. 16.3).

Aus diesen Überlegungen heraus haben May und Keller [9] drei Formen des auftretenden Verkehrs charakterisiert:

- den freien Verkehr mit hohen Geschwindigkeiten und geringen Verkehrsstärken und -dichten,

- den teilgebundenen Verkehr, bis zu dem Bereich maximaler Verkehrsstärken, optimaler Geschwindigkeit und Verkehrsdichte,

- den gebundenen Verkehr mit hohen Verkehrsdichten, geringen Verkehrsstärken und Geschwindigkeiten.

\subsubsection{Kapazität und Stabilität}

Die Effizienz des Verkehrssystems ist abhängig von der Kapazität einer Verkehrsanlage, die als die ,größte Verkehrsstärke, die ein Verkehrsstrom bei gegebenen Weg- und Verkehrsbedingungen an dem für ihn bestimmten Querschnitt erreichen kann“ definiert ist [3]. Die Kapazität bestimmt sich durch die Dichte der Fahrzeugkolonne und die Geschwindigkeit, mit der die Fahrzeugkolonne den Querschnitt passiert.

Die Verkehrsdichte wird durch die Abstände zwischen den Fahrzeugen festgelegt. Dabei gilt die Faustregel, dass der Sicherheitsabstand in Metern, den ein Fahrer zum voraus- 


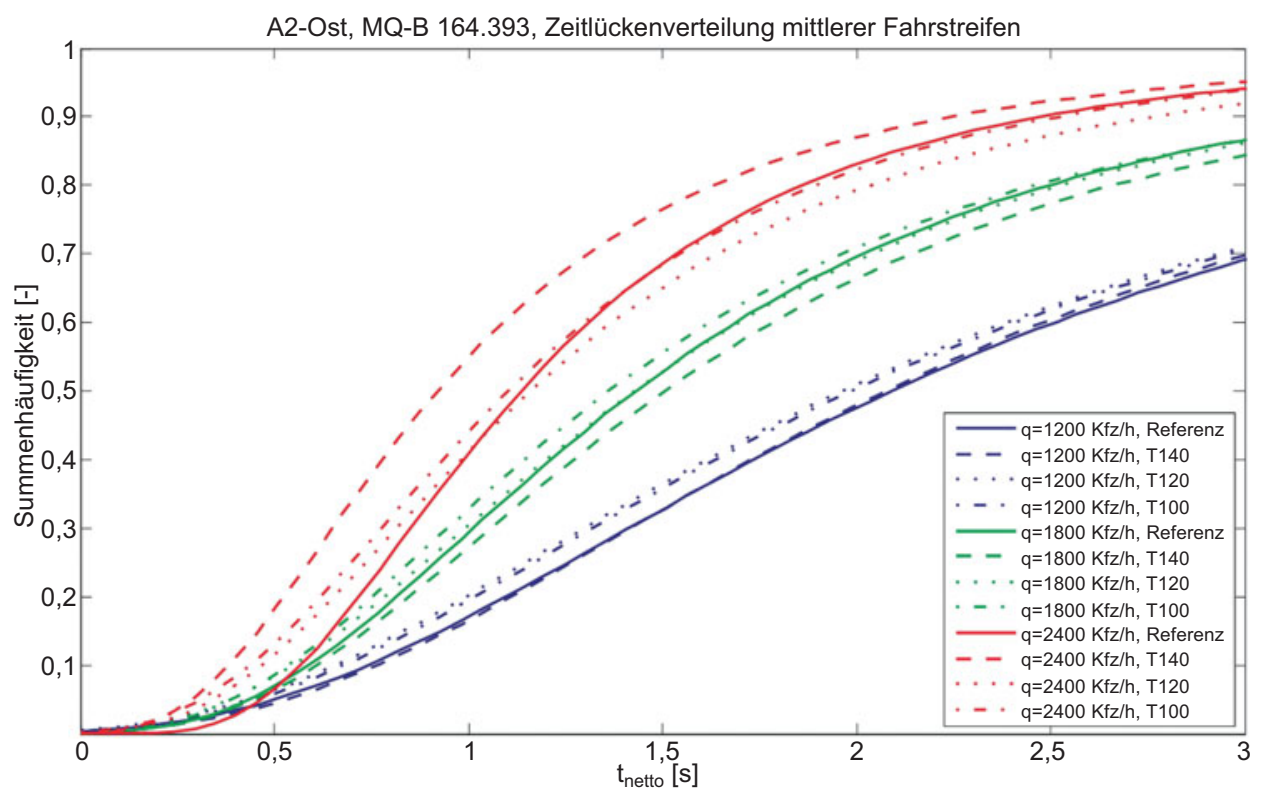

Abb. 16.4 Verteilungsfunktionen der Zeitlückenverteilung für unterschiedliche Verkehrsnachfragen und Geschwindigkeitsbeschränkungen [2]

fahrenden Fahrzeug einhalten soll, den halben Wert der aktuellen Geschwindigkeit in Kilometern pro Stunde beträgt. Diese allgemein bekannte Regel des „halben Tachoabstands" geht von einer Reaktionszeit aus, die kleiner als 1,8 $\mathrm{s}$ ist, da bei diesem Wert und gleichbleibender Geschwindigkeit genau der Abstand zum vorausfahrenden Fahrzeug zurückgelegt wird. Dieser Mindestabstand wird auch üblicherweise in der Rechtsprechung gefordert (s. z. B. [7]). Für Lkw schreibt die Straßenverkehrsordnung bei Geschwindigkeiten über $50 \mathrm{~km} / \mathrm{h}$ ausdrücklich einen Mindest-Wegabstand von $50 \mathrm{~m}$ vor, der bei der zulässigen Höchstgeschwindigkeit für Fahrzeuge über 7,5 t auf Autobahnen eine Zeitlücke von 2,25 s erfordert.

Ausgehend von einer Reaktionszeit von 1,8 s kann in einem einfachen Ansatz die Kapazität eines Fahrstreifens also mit etwa 2000 Fahrzeugen pro Stunde angesetzt werden. Dies gilt gleicherweise für Stadtstraßen wie für Landstraßen oder Autobahnen. Empirische Untersuchungen zeigen allerdings, dass die Zeitlücken im Mittel deutlich unterhalb der $1,8 s$ liegen und insbesondere bei hohen Verkehrsstärken Werte um die 1,0 s einnehmen. Die $15 \%$-Perzentile der Verteilung liegt in diesen Fällen sogar bei Werten unter $0,5 \mathrm{~s}$ (s. auch Abb. 16.3). Dies bedeutet, dass $15 \%$ der Fahrzeuge mit Zeitlücken, die kleiner als $0,5 s$ sind, auf das vorausfahrende Fahrzeug folgen. Abb. 16.4 zeigt für unterschiedliche Verkehrsstärkebereiche und unterschiedliche Geschwindigkeitsbeschränkungen die korrespondierenden Zeitlückenverteilungen.

Aufgrund der geringen Folgeabstände bei relativ hohen Geschwindigkeiten werden in empirischen Untersuchungen auch Kapazitäten ermittelt, die deutlich über den genannten 


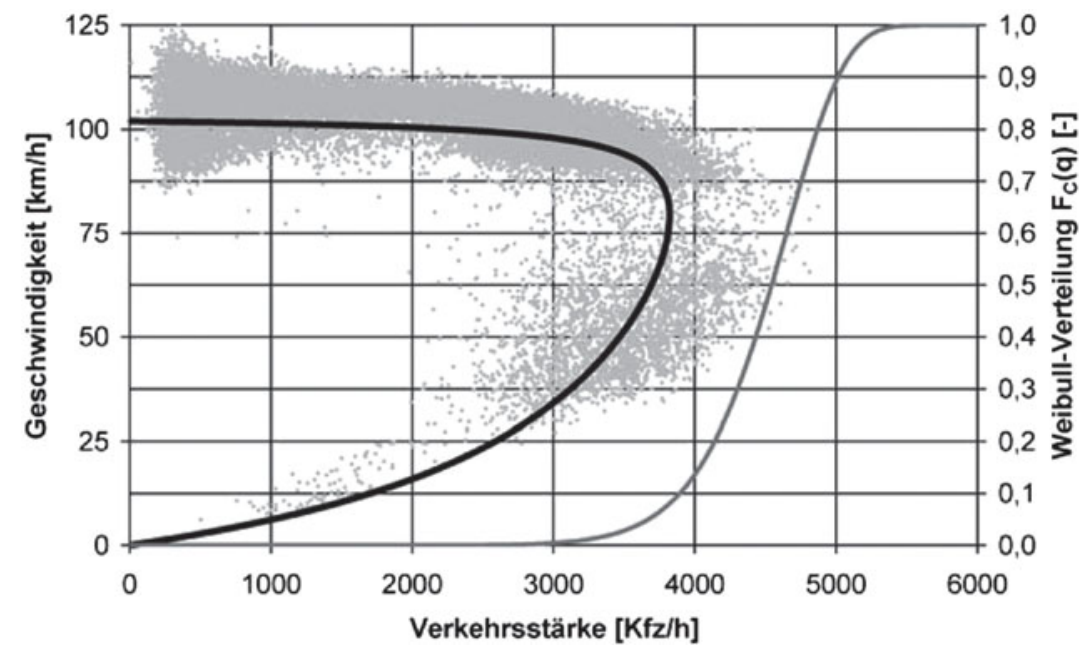

Abb. 16.5 Messwerte für Fünf-Minuten-Intervalle im q-v-Diagramm und die zugehörige Kapazitätsverteilung für einen zweistreifigen Autobahnquerschnitt [1]

$2000 \mathrm{Kfz} / \mathrm{h}$ liegen können. Ferner zeigen diese Untersuchungen, dass es keinen exakten Wert gibt, bis zu dem der Verkehrsfluss immer stabil verläuft und genau dann zusammenbricht, wenn dieser Wert überschritten wird. Vielmehr ist zu beobachten, dass die Kapazität eine Zufallsgröße darstellt, die durch eine Verteilung dargestellt werden kann. Untersuchungen [1] an vielen Streckenabschnitten zeigen, dass Kapazitäten von Autobahnen typischerweise Weibull-verteilt sind und z. B. für dreistreifige Richtungsfahrbahnen eine Standardabweichung von ca. $600 \mathrm{Kfz} / \mathrm{h}$ (gemessen in Fünf-Minuten-Intervallen) und damit eine unerwartet breite Variabilität aufweisen.

Der Erwartungswert der Kapazität entspricht in dieser stochastischen Sichtweise einer Nennkapazität und stellt das 50\%-Perzentil der Verkehrsstärkewerte dar, die Ausgangspunkt eines Zusammenbruchs waren. Je näher die Verkehrsbelastung an dieser Nennkapazität bzw. darüber liegt, umso höher wird die Wahrscheinlichkeit des Zusammenbruchs und eines Staus.

Der Zusammenbruch führt in allen Fällen über den Zustand des synchronisierten Verkehrs zum Stau, und auch die Erholung verläuft über den synchronisierten Verkehr zurück zu einem stabilen Verkehrsablauf mit höheren Geschwindigkeiten (s. Abb. 16.6). Die Verkehrsstärke geht in den Übergängen zum synchronisierten bzw. zum gestauten Verkehr jeweils zurück, und eine Erholung erfolgt auf einem niedrigeren Niveau. Dieser Effekt des capacity drop wird dadurch verursacht, dass die Fahrer beim Verlassen der stromabwärtigen Staufront einen größeren Abstand einhalten als zuvor im fließenden Verkehr vor dem Zusammenbruch.

Nach Hall und Agyemang-Duah [6] liegt dieser capacity drop bei 5-6\%, Untersuchungen von Brilon und Ponzlet [10] auf deutschen Autobahnen ergaben Werte zwischen 4 und $12 \%$. 


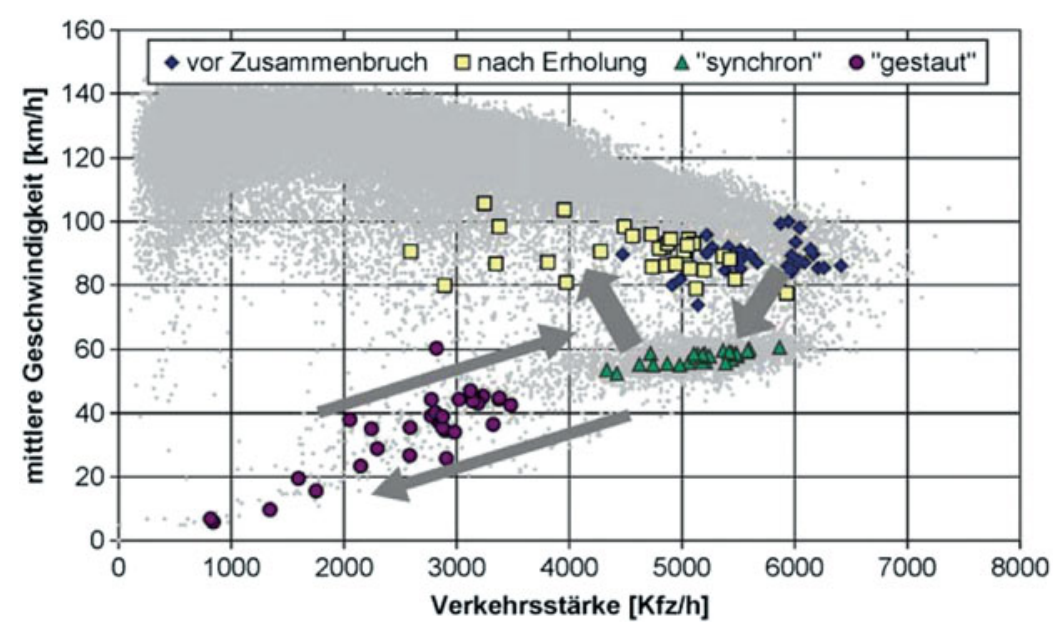

Abb. 16.6 Muster der Verkehrsdynamik mit den Übergängen zwischen den Zuständen des stabilen in den synchronen und gestauten Verkehr. Die Werte wurden an einer dreistreifigen Fahrbahn in Fünf-Minuten-Intervallen gemessen [1]

\subsection{Verkehrliche Wirkung autonomer Fahrzeuge}

Die Effizienz des Verkehrssystems ist abhängig von der Kapazität einer Verkehrsanlage. Bei einer Nutzung der Verkehrsanlage durch autonom fahrende Fahrzeuge wird sich die Kapazität gegenüber einer Nutzung durch menschliche Fahrer verändern. Für den Verkehrsfluss sind entweder die Kapazitäten der Streckenabschnitte oder der Knotenpunkte maßgebend. Während für die Leistungsfähigkeit städtischer Straßennetze vor allem die Kapazitäten der Knotenpunkte und dort der Lichtsignalanlagen relevant sind, sind auf Autobahnen auch die Kapazitäten freier Strecken ausschlaggebend. Aus diesem Grund betrachten die folgenden Überlegungen die Kapazitäten für beide Fälle unter Berücksichtigung, dass ein noch nicht bekannter Anteil der Fahrzeuge autonom fährt.

\subsubsection{Streckenabschnitte von Autobahnen}

\subsubsection{Kapazität}

Die Kapazität eines Fahrstreifens ist festgelegt durch die maximale Anzahl von Fahrzeugen, die einen Querschnitt während einer Zeiteinheit passieren können. Sie bestimmt sich durch die Dichte der Fahrzeugkolonne und der Geschwindigkeit, mit der die Fahrzeugkolonne den Querschnitt passiert. Die Zustandsgleichung, die das Verhältnis dieser grundlegenden Kenngrößen des Verkehrsablaufs beschreibt, lautet:

$$
q=k \cdot v(k)
$$


In einem homogenen Verkehrsfluss ist die Dichte einfach zu bestimmen und ergibt sich aus dem Reziprok des Platzbedarfs eines Fahrzeugs [11]:

$$
k=\frac{1}{v T_{h}+L} .
$$

In diesem Zusammenhang ist $T_{h}$ der zeitliche Abstand (Zeitlücke) zum vorausfahrenden Fahrzeug und $L$ die Länge eines Fahrzeugs. Da die Kapazität die maximale Verkehrsstärke $q_{\text {max }}$ darstellt, ist diese folglich eine Funktion von $v, T_{h}$ und $L$. Für den Fall, dass ausschließlich menschliche Fahrer die Fahrzeuge steuern, ergibt sich die Kapazität $C_{h}$ mit:

$$
C_{h}=q_{\max }=\frac{v}{v T_{h}+L} .
$$

Analog wird die Kapazität $C_{a}$ in einem rein durch autonome Fahrzeuge zusammengesetzten Verkehrsfluss durch die folgende Funktion beschrieben, in der $T_{a}$ die von autonomen Fahrzeugen bevorzugte Zeitlücke darstellt:

$$
C_{a}=\frac{v}{v T_{a}+L} .
$$

Das Verhältnis der beiden Kapazitätswerte und damit die Veränderung der Kapazität wird durch den Zusammenhang

$$
C_{a} / C_{h}=\left(v T_{h}+L\right) /\left(v T_{a}+L\right)
$$

beschrieben.

Für die Abschätzung des Einflusses autonomer Fahrzeuge auf die Kapazität werden für die Parameter der Kapazitätsformel Größen verwendet, die für heutige Verhältnisse empirisch nachgewiesen sind. So erscheint es sinnvoll als mittlere Geschwindigkeit, bei der die Kapazität erreicht wird, den Wert $v=80 \mathrm{~km} / \mathrm{h}(22, \overline{2} \mathrm{~m} / \mathrm{s})$ anzunehmen. Für den Platzbedarf eines mittleren Personenkraftwagens wird das bekannte Maß einer mittleren Fahrzeuglänge von 4,5 $m$ und eines minimalen Sicherheitsabstands zum Vordermann von 3,0 $m$ und somit $L_{P k w}=7,5 m$ verwendet. Als mittlere Länge eines Lastkraftwagens werden $18 m$ als gewichtetes Mittel der Längen eines Lastzuges $(18,75 \mathrm{~m}$ ) und eines Sattelschleppers $(16,50 \mathrm{~m})$ angesetzt. Für den Platzbedarf eines Lastkraftwagens ergeben sich unter Berücksichtigung von 3,0 $\mathrm{m}$ Abstand zum vorausfahrenden Fahrzeug $L_{L k w}=21 \mathrm{~m}$. Als realistische Größe für den mittleren zeitlichen Folgeabstand bei hohen Verkehrsstärken liefern die empirischen Untersuchungen einen Wert von $T_{h}=1,15 \mathrm{~s}$.

Für die Veränderung der Kapazität bei autonomem Fahren ist die veränderte Folgezeitlücke $T_{a}$ ausschlaggebend. Als technisch realisierbar und zugleich aus Sicht der Verkehrsteilnehmer akzeptabel erscheint hierfür ein Wert von $T_{a}=0,5 \mathrm{~s}$. Dieser sehr kurze Folgeab- 


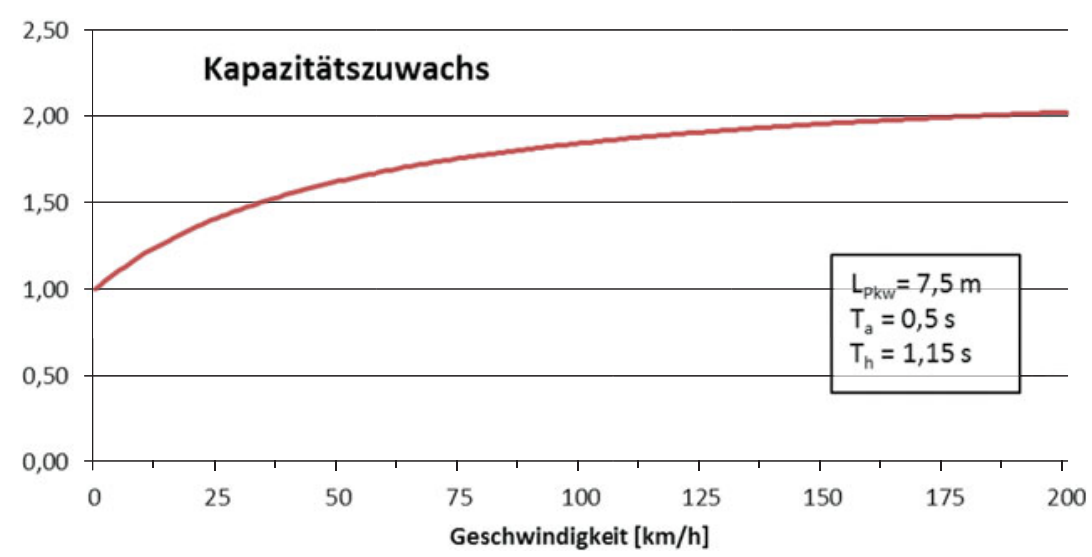

Abb. 16.7 Kapazitätszuwachs, der sich bei einer ausschließlich autonomen Fahrzeugflotte (nur Pkw) in Abhängigkeit der Geschwindigkeit ergeben würde

stand tritt bereits heute in Abhängigkeit der Verkehrsverhältnisse bei bis zu $20 \%$ aller Folgeabstände auf. Insofern scheint dieser Abstand akzeptabel, sofern aus technischer Sicht die Sicherheit gewährleistet ist.

Für die unterstellten Größen würde sich bei Verwendung der oben hergeleiteten Formeln die Kapazität und damit der maximale Durchfluss im Falle eines rein autonomen Verkehrs deutlich erhöhen (Faktor 1,78) (s. Abb. 16.7).

Gegenüber den heute beobachteten Kapazitätswerten eines Fahrstreifens von $2200 \mathrm{Kfz} / \mathrm{h}$ wäre damit bei einem rein autonomen Verkehr eine Steigerung der Verkehrsstärke auf etwa $3900 \mathrm{Kfz} / \mathrm{h}$ möglich.

Wird der Schwerverkehr im Verkehrsfluss berücksichtigt, so kann der mittlere Platzbedarf der Fahrzeuge aus einer mit dem Schwerverkehrsanteil $\omega$ gewichteten Summe gebildet werden. Die Verkehrsdichte erhält man dann wiederum aus dem Reziprok des mittleren Platzbedarfs eines Fahrzeugs mit

$$
k=\frac{1}{(1-\omega)\left(v T_{h}+L_{P k w}\right)+\omega\left(v T_{h}+L_{L k w}\right)} .
$$

Für die Kapazität ergibt sich bei dieser Betrachtung der Zusammenhang

$$
\mathrm{C}=\frac{v}{(1-\omega)\left(v T_{a}+L_{P k w}\right)+\omega\left(v T_{a}+L_{L k w}\right)} .
$$

Geht man von einer für den autonomen Verkehr auf Autobahnen moderaten Geschwindigkeit von $80 \mathrm{~km} / \mathrm{h}$ aus, so erhält man den im folgenden Diagramm dargestellten funktionalen Zusammenhang. Für einen auf deutschen Autobahnen typischen Lkw-Anteil von 15\% würde sich dann eine Kapazität von etwa $3877 \mathrm{Kfz} / \mathrm{h}$ und damit nahezu der doppelte Wert 


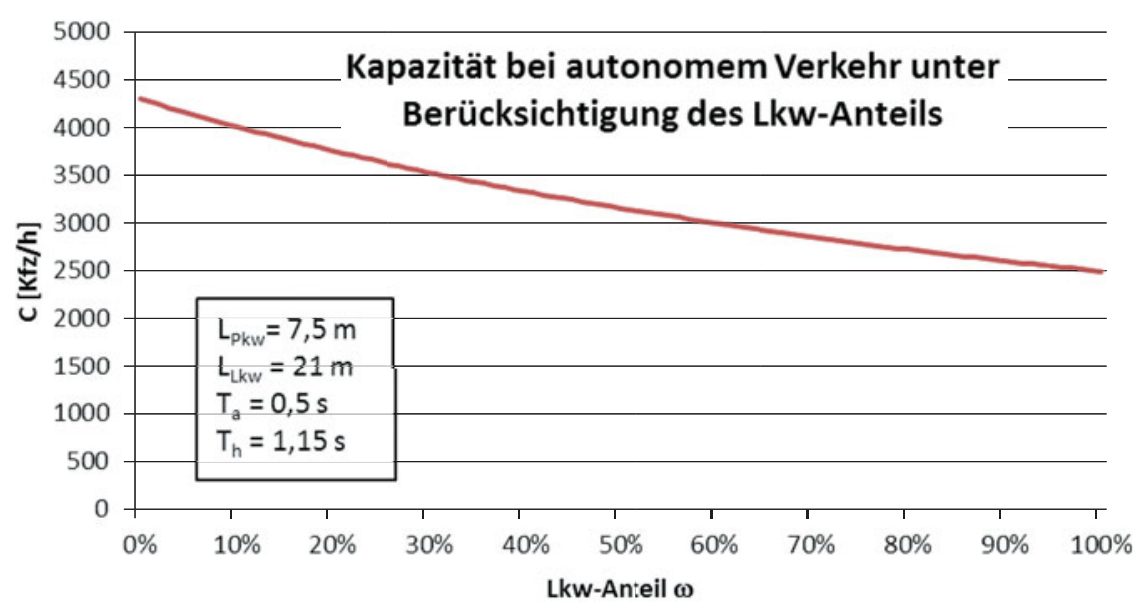

Abb. 16.8 Kapazität eines Fahrstreifens bei rein autonomem Verkehr in Abhängigkeit des LkwAnteils

gegenüber heute empirisch nachgewiesenen Kapazitäten einstellen. Setzt man in die Kapazitätsformel für eine Plausibilitätsprüfung $T_{a}=1,15 \mathrm{~s}$ bei ansonsten unveränderten Parametern, ergibt sich bei einem Schwerverkehrsanteil von $15 \%$ eine Kapazität von etwa $2280 \mathrm{Kfz} / \mathrm{h}$. Dieser Wert entspricht den bei heutigen Verhältnissen gemessenen Kapazitäten und bestätigt die richtige Wahl des Rechenansatzes sowie der Parameter.

Bei gemischtem Verkehr, in dem autonome Fahrzeuge mit einem Anteil von $\eta$ am Gesamtaufkommen vertreten sind, ist die Kapazität $C_{m}$ zusätzlich von der Anteilsgröße $\eta$ abhängig:

$$
\mathrm{C}_{m}=\frac{v}{\eta v T_{a}+(1-\eta) v T_{h}+L_{P k w}} .
$$

Setzt man auch hier wiederum mit $v=80 \mathrm{~km} / \mathrm{h}$ und $L_{P k w}=7,5 \mathrm{~m}$ realistische Größen in die Gleichung ein, ergibt sich der im folgenden Diagramm dargestellte Zusammenhang (s. Abb. 16.9). Aus der Abbildung wird deutlich, dass die Kapazität bei geringeren Ausstattungsraten unterproportional anwächst und bei $\eta=0,5$ erst einen Wert von etwa $3100 \mathrm{Pkw} / \mathrm{h}$ und damit von $36 \%$ der möglichen Steigerung bei Vollausstattung erreicht.

Berücksichtigt man darüber hinaus, dass autonome Fahrzeuge einen zusätzlichen Abstand auf ein von einem menschlichen Fahrer gelenktes Fahrzeug lassen sollten, um diesen Fahrer nicht zu bedrängen, wird die Bestimmung der Kapazität etwas komplizierter. In dieser Betrachtung müssen die Kombinationen aufeinanderfolgender Fahrzeuge (a-a, a-h, h-a, und h-h) und die zugehörigen Zeitlücken $\left(T_{a a}, T_{a h}, T_{h x}\right)$ betrachtet werden, um zu einer modifizierten Kapazitätsgleichung zu gelangen:

$$
\mathrm{C}_{m}=\frac{v}{\eta^{2} v T_{a a}+\eta(1-\eta) v T_{a h}+(1-\eta) v T_{h x}+L} .
$$




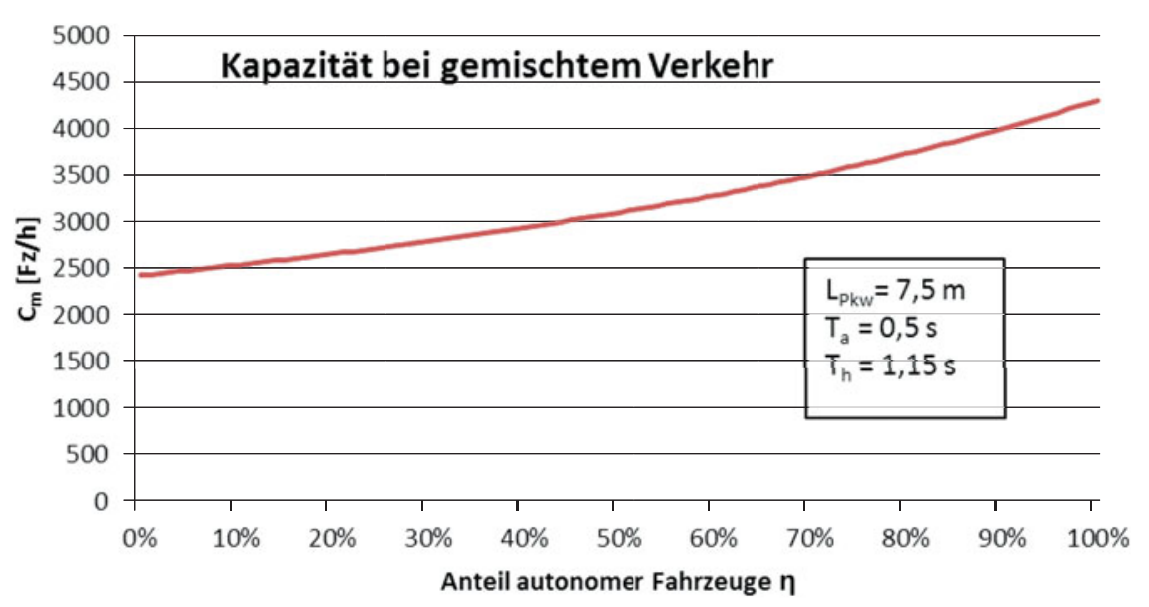

Abb. 16.9 Kapazität eines Fahrstreifens in Abhängigkeit des Anteils autonomer Fahrzeuge bei reinem Pkw-Verkehr

Als realistische Werte können für die Zeitlückendauern die Werte $T_{a a}=0,5 \mathrm{~s}, T_{a h}=0,9 \mathrm{~s}$, $T_{h x}=1,15 s$ angenommen werden. In dieser Betrachtung steigt die Kapazität in Abhängigkeit der Ausstattungsrate im unteren Bereich noch etwas langsamer an und erreicht für $\eta=0,5$ einen Wert von $2850 \mathrm{Kfz} / \mathrm{h}$, um bei einer Ausstattung von $100 \%$ den Kapazitätswert von knapp $4300 \mathrm{Kfz} / \mathrm{h}$ zu erreichen (s. Abb. 16.10).

Dieselbe Vorgehensweise kann für die Abschätzung der Kapazität für reinen LkwVerkehr angewendet werden, der auf einem Fahrstreifen einer Autobahn organisiert werden könnte. Bei unveränderten Annahmen für die erforderlichen Zeitlücken wird der Platzbedarf wiederum mit $L=21 \mathrm{~m}$ gewählt. Mit diesen Eingangswerten ergibt sich bei rein autonomem Fahren ein Kapazitätswert von $2420 \mathrm{Lkw} / \mathrm{h}$ anstatt der bei menschlichen Fahrern erreichbaren $1720 \mathrm{Lkw} / \mathrm{h}$.

\subsubsection{Stabilität}

Neben der Kapazität, die die größte Verkehrsstärke darstellt, die ein Verkehrsstrom bei gegebenen Weg- und Verkehrsbedingungen an dem für ihn bestimmten Querschnitt erreichen kann, ist insbesondere die Stabilität des Verkehrsablaufs für die Effizienz von Bedeutung. Deutlich wird dies, wenn die Kapazität als stochastische Größe betrachtet wird, die die Wahrscheinlichkeit des Zusammenbruchs in Abhängigkeit der Verkehrsstärke wiedergibt. Je größer die Standardabweichung in der Wahrscheinlichkeitsverteilung ist, umso größer ist die Wahrscheinlichkeit des Zusammenbruchs bereits bei geringeren Verkehrsstärken und damit die Instabilität.

Kommt es zum Zusammenbruch, vermindert sich durch den Effekt des capacity drop die Kapazität spürbar in der in der o.g. Literatur [10] angegebenen Größenordnung von etwa $10 \%$.

Verschiedene orts- und zeitabhängige Faktoren führen bei gleicher Fahrstreifenanzahl und gleichen verkehrlichen Randbedingungen (Verkehrsstärke, Schwerverkehrsanteil) zu 


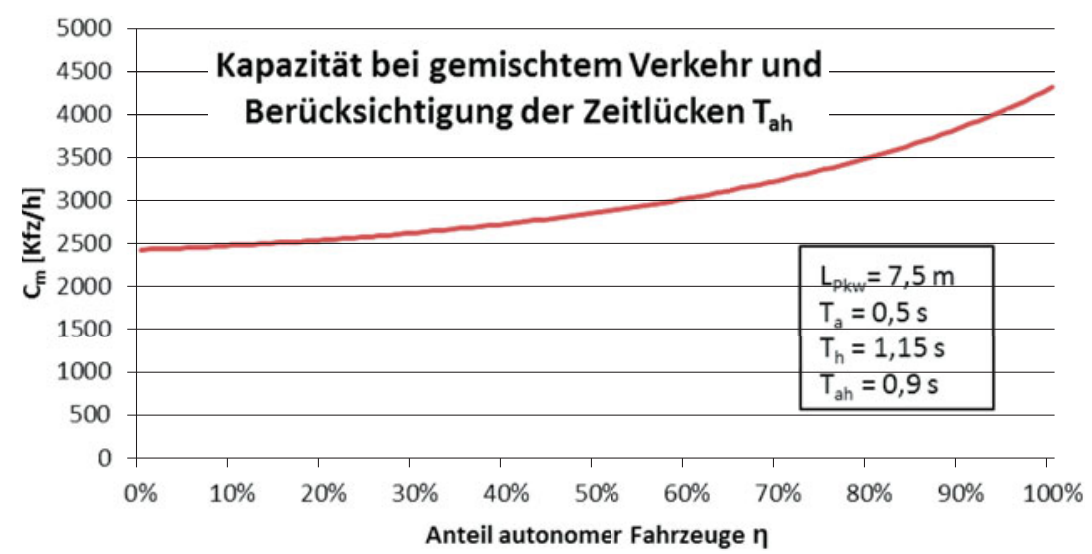

Abb. 16.10 Kapazität eines Fahrstreifens in Abhängigkeit des Anteils autonomer Fahrzeuge bei reinem Pkw-Verkehr unter Berücksichtigung größerer Folgezeitlücke von autonomen Fahrzeugen auf von Fahrern gelenkte Fahrzeuge

unterschiedlichen Verteilungsfunktionen der Kapazität. Wesentliche Einflussfaktoren sind in diesem Zusammenhang die Geschwindigkeits- und die Zeitlückenverteilungen. Je geringer die Standardabweichungen sind, umso stabiler ist der Verkehrsablauf und umso weniger Zusammenbrüche sind auch bei hohen Verkehrsstärken zu erwarten.

Autonome Fahrzeuge werden, insbesondere wenn sie durch Kommunikation untereinander die Aktionen der vorausfahrenden Fahrzeuge antizipieren können, zu einer Verstetigung des Verkehrsablaufs und somit zur Stabilität beitragen. Bei einem rein autonomen Verkehr ist davon auszugehen, dass eine vollständige Stabilität erreicht wird und ein Kapazitätsabfall vermieden werden kann.

\subsubsection{Knotenpunkte mit Lichtsignalanlage}

Da Knotenpunkte im Zuge höher belasteter Straßen in der Regel mit Lichtsignalanlagen geregelt werden, beziehen sich die folgenden Überlegungen auf die Kapazitäten von Knotenpunkten mit Lichtsignalsteuerung.

Bei hoher Verkehrsnachfrage kommt es an signalisierten Knotenpunkten unabhängig von der Koordinierung der Lichtsignalanlagen zu einem ständigen Rückstau. Deshalb erfolgt die Anfahrt der wartenden Fahrzeugkolonne bei Beginn der Freigabezeit regelmäßig aus dem Stand. Wenn das erste Fahrzeug bei Beginn der Freigabe angefahren ist, folgt das nächste, wenn ein bestimmter zeitlicher Abstand erreicht ist. Dieser zeitliche Abstand wird durch den Zeitbedarfswert repräsentiert, der für Standardbedingungen (keine Längsneigung, Geradeausverkehr, ausreichende Fahrstreifenbreite) und reinen Pkw-Verkehr $t_{b}=1,8 \mathrm{~s}$ beträgt [4]. Dies entspricht einer Sättigungsverkehrsstärke von $q_{s}=2000 P k w / h$. Für Lkw und Busse werden als Zeitbedarfswert $t_{b}=3,15 \mathrm{~s}$ und für Lastzüge $t_{b}=4,5 \mathrm{~s}$ angegeben. 
Im Kolonnenstart kann der Anfahrzeitpunkt aufgrund der Bewegung der vorausfahrenden Fahrzeuge antizipiert werden. Dadurch reduziert sich die Reaktionszeit auf das Losfahren des direkten Vordermanns und kann mit $T_{h}=0,6 \mathrm{~s}$ angenommen werden. Für einen mittleren Platzbedarf eines Pkw im Rückstau vor einer Lichtsignalanlage von 7,5 $m$ (bei einer Fahrzeuglänge von 4,5 $m$ und einem Abstand von Stoßstange zu Stoßstange von 3,0 $\mathrm{m}$ ) sowie plausiblen Werten für die durchschnittliche Geschwindigkeit $v=22,5 \mathrm{~km} / \mathrm{h}$ an der Haltlinie der Lichtsignalanlage erhält man über den Zusammenhang $t_{b}=T_{h}+L / v=$ $1,8 s$ eine Bestätigung des Zeitbedarfswerts. Dies gilt in gleicher Weise für die Zeitbedarfswerte von Lkw und Lastzügen mit einer Länge von $12 \mathrm{~m}$ bzw. $18 \mathrm{~m}$. Folglich kann die Sättigungsverkehrsstärke eines Fahrstreifens an einer Lichtsignalanlage mit der Zustandsgleichung angegeben werden:

$$
q_{s}=\frac{v}{v T_{h}+L} .
$$

Für die Betrachtung der Sättigungsverkehrsstärken rein autonomem und gemischten Verkehrs gelten die Zusammenhänge, die für die Kapazität von Streckenabschnitten abgeleitet wurden mit den hier für den Kolonnenstart an Lichtsignalanlagen unterstellten Werten von $v=22,5 \mathrm{~km} / \mathrm{h}=6,25 \mathrm{~m} / \mathrm{s}, T_{h}=0,6 \mathrm{~s}$. Für das autonome Fahren wird unterstellt, dass die Reaktionszeit bzw. der Sicherheitsabstand auch im dichten und langsamen Stadtverkehr folgende Werte nicht unterschreiten soll: $T_{a}=0,3 \mathrm{~s}, T_{a a}=0,3 \mathrm{~s}, T_{a h}=0,6 \mathrm{~s}, T_{h x}=0,6 \mathrm{~s}$.

Die Kapazität eines Fahrstreifens an Knotenpunkten mit Lichtsignalanlage wird einerseits von den Sättigungsverkehrsstärken und andererseits von der zur Verfügung stehenden Freigabezeit bestimmt. Die Freigabezeiten, die während einer Stunde den verschiedenen Verkehrsströmen zur Verfügung gestellt werden können, sind ihrerseits abhängig von der Umlaufzeit und den Zwischenzeiten. In den Hauptverkehrszeiten wird meist eine Umlaufzeit von $90 s$ gewählt, sodass während einer Stunde die Zwischenzeiten 40-mal berücksichtigt werden müssen. Geht man von einem typischen, im Zuge einer städtischen Hauptverkehrsstraße gelegenen Knotenpunkt aus, so kommt für diesen häufig ein dreiphasiges Signalprogramm zum Einsatz. Die für eine Hauptrichtung relevanten Zwischenzeiten in den drei Phasenübergängen summieren sich in der Größenordnung auf $20 s$ und sind im Wesentlichen von den Räumzeiten der quer laufenden Fußgänger abhängig. Bei einer Umlaufzeit von $90 s$ verbleiben dann noch $70 s$ für die Freigabezeiten der verschiedenen Ströme. Unterstellt man, dass von der verbleibenden Freigabezeit 50 \% für die Ströme der Hauptrichtung zur Verfügung stehen, ergibt sich während einer Stunde eine Freigabezeit von $1400 s$ bzw. ein Freigabezeitanteil von $p_{F}=38,89 \%$, also ein Anteil, der in der Größenordnung von etwa 40 \% liegt.

Bei konfliktfreier Signalisierung (d.h. keine bedingten Verträglichkeiten z. B. mit parallel geführten Fußgängern) kann vor diesem Hintergrund die Kapazität von gemischten Verkehrsströmen mit dem o.g. Ansatz ermittelt werden:

$$
C_{L S A}=q_{s} \cdot p_{F}=\frac{v \cdot p_{F}}{v T_{h}+L}
$$


Für heutige Verhältnisse mit ausschließlich durch Menschen gesteuerten Fahrzeugen ergibt sich damit bei Verwendung der o. g. Werte eine Kapazität etwa $800 P k w / h$ je Fahrstreifen. Bei einem rein autonomen Verkehr mit $T_{a}=0,3 \mathrm{~s}$ würde sich die Kapazität auf etwa $1120 \mathrm{Pkw} / \mathrm{h}$ und damit um etwa 40 \% erhöhen. Für eine gemischte Zusammensetzung der Verkehrsströme liegen die Effizienzgewinne zwischen diesen angegebenen Werten und können mit den oben eingeführten Formeln bestimmt werden.

Die Formel macht außerdem deutlich, dass neben der Dauer der Folgezeitlücken vor allem die Geschwindigkeit von Bedeutung für die Kapazität ist. Mit steigender Räumgeschwindigkeit wächst die Kapazität bei autonomem Verkehr überproportional gegenüber einem Verkehr mit Menschen als Fahrern (s. Abb. 16.11). Gelingt es also, beim autonomen Fahren neben den kürzeren Zeitlücken auch ein zügigeres Anfahren und Räumen zu erreichen, ist damit ein deutlich höherer Kapazitätsgewinn als die oben angegebenen $40 \%$ zu erwarten.

\subsubsection{Abschätzung der Effizienzgewinne durch autonomes Fahren}

Die Abschätzungen der Wirkung autonomer Fahrzeuge auf die Kapazität als Maß für die Effizienz von Verkehrsanlagen zeigen signifikante Steigerungspotenziale sowohl für die Streckenabschnitte von Fernstraßen als auch für die Knotenpunkte im Zuge städtischer Hauptverkehrsstraßen.

Im Stadtverkehr könnte bei rein autonomem Verkehr eine Kapazitätserhöhung von etwa $40 \%$ erreicht werden, während die Kapazitäten auf Autobahnabschnitten um etwa 80\% gesteigert werden könnten. Der deutliche Unterschied in den Steigerungspotenzialen ist in der durchschnittlichen Geschwindigkeit, mit der die Verkehrsanlagen befahren werden, begründet. Dies wird aus dem Verlauf des Funktionsgraphen in Abb. 16.7 deutlich, der einen überproportionalen Anstieg der Kapazität im Bereich geringerer Geschwindigkeiten zeigt und hin zu höheren Geschwindigkeiten abflacht. Bei Erreichen der Kapazität liegen die Geschwindigkeiten auf Autobahnen bei etwa $80 \mathrm{~km} / \mathrm{h}$. Auf städtischen Hauptverkehrsstraßen ergibt sich beim für die Kapazität maßgebenden Kolonnenstart an Lichtsignalanlagen eine durchschnittliche Geschwindigkeit von etwa $20 \mathrm{~km} / \mathrm{h}$. Wegen dieses Unterschieds in den Geschwindigkeiten wirken sich autonome Fahrzeuge sehr unterschiedlich auf die Kapazität der Verkehrsanlagen aus.

Neben dem Kapazitätswert, der unter Berücksichtigung autonomer Fahrzeuge erreicht werden kann, ist insbesondere die Stabilität des Verkehrsablaufs bei hohen Verkehrsstärken von Bedeutung. Im Stadtverkehr kommt es zwar ab einer Auslastung von 70\%-80 \% zu einem ständigen Rückstau vor den maßgebenden Lichtsignalanlagen, weshalb bei hohen Belastungen eine Durchfahrt ohne Halt (grüne Welle) nicht mehr möglich ist, aber es entsteht kein Leistungsabfall (capacity drop), wie dies im Außerortsverkehr und vor allem auf Autobahnen der Fall ist. Insofern ist die Stabilität des Verkehrsablaufs im städtischen Straßennetz bis zum Erreichen der Kapazität nicht gefährdet und erst bei einer Überlastung kommt es zu Blockaden in den Knotenpunkten. 


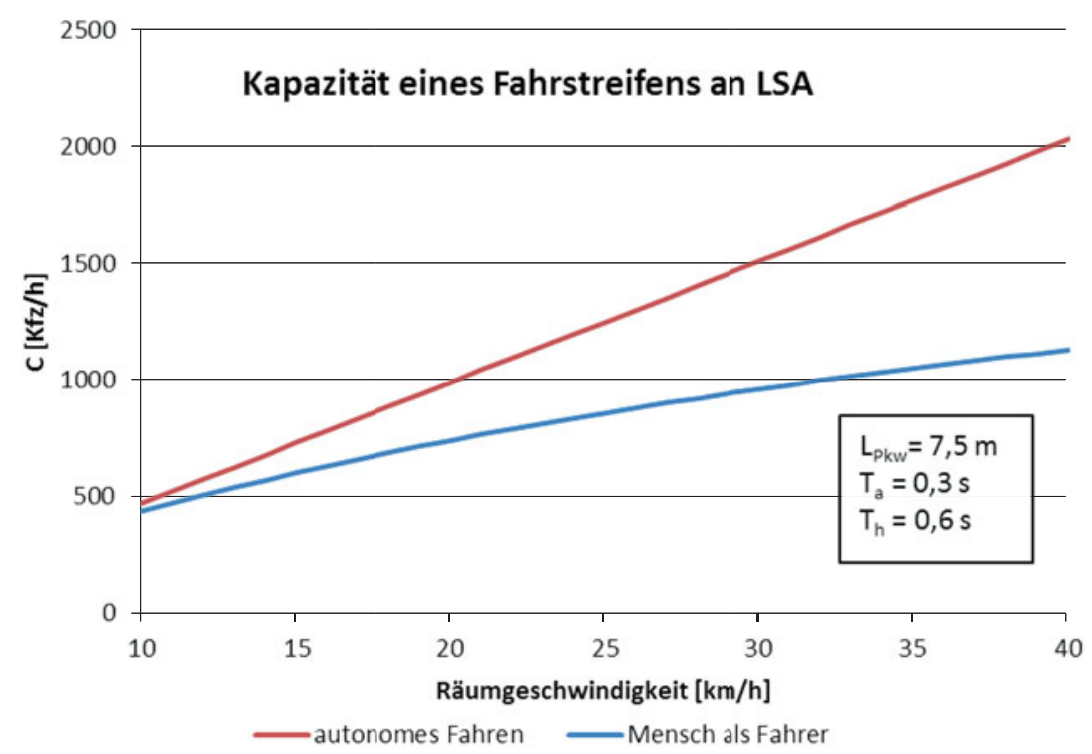

Abb. 16.11 Kapazitätswerte für einen Fahrstreifen an einer Lichtsignalanlage in Abhängigkeit der Räumgeschwindigkeit

\subsection{Zusammenfassung und Ausblick}

\subsubsection{Verkehr}

Die Überlegungen mithilfe der makroskopischen Modelle des Verkehrsflusses zeigen, dass durch autonome Fahrzeuge grundsätzlich eine signifikante Kapazitätssteigerung zu erwarten ist, wodurch bestehende Verkehrsinfrastrukturen effizienter genutzt werden könnten. In Verbindung mit der erwarteten Steigerung der Kapazität für bestehende Verkehrsinfrastrukturen reduzieren sich Stauungen und Verlustzeiten, wodurch in der Folge die Qualität des Verkehrsablaufs erhöht wird. Insbesondere zwei Effekte bewirken die Kapazitätssteigerung:

1. Ein Effekt ist die Reduktion der Zeitlücken zwischen autonomen Fahrzeugen. In diesem Zusammenhang erscheint es bedeutsam, dass der Fahrkomfort trotz kurzer Zeitlücken durch die Antizipation der Aktionen der vorausfahrenden Fahrzeuge und der dadurch ermöglichten geringeren Beschleunigungs- bzw. Verzögerungswerte gesichert wird. Dies könnte auch für die Kolonnenstabilität von Bedeutung sein. Für die Antizipation erscheint die Kommunikation zwischen den Fahrzeugen und auch zwischen Fahrzeugen und der Infrastruktur eine wichtige Voraussetzung zu sein.

2. Neben der Zeitlückendauer ist die Geschwindigkeit des Fahrzeugpulks von großer Bedeutung. Je höher die Geschwindigkeit bei konstanter Dichte ist, umso höher ist die Verkehrsstärke an einem Querschnitt. Hohe Geschwindigkeiten bei gleichbleibender 
Verkehrsdichte sind jedoch nur im rein autonomen Verkehr möglich. Bereits ein von einem Menschen gelenktes Fahrzeug würde in der Kolonne zu langsameren Geschwindigkeiten führen und den Kapazitätsgewinn reduzieren.

\subsubsection{Infrastruktur}

Die entwickelten Modelle für den Verkehrsfluss und die Kapazität unter Berücksichtigung eines Anteils autonomer Fahrzeuge zeigen, dass die Kapazität mit dem Anteil autonomer Fahrzeuge überproportional anwächst. Dabei ist zu bemerken, dass die Verkürzung der Zeitlücken schon ab dem ersten autonomen Fahrzeug wirkt, die Steigerung der Geschwindigkeit bei hohen Dichten hingegen nur für rein autonomen Verkehr und damit bei höheren Ausstattungsraten möglich sein wird.

Die Einführung autonomer Fahrzeuge wird nach Meinung des Autors nur über deren Fähigkeit gelingen, sich im gemischten Verkehr sicher zu bewegen, da reservierte Bewegungsflächen gerade bei geringen Ausstattungsraten weder ökonomisch noch sozial zu vertreten wären. Sobald jedoch eine ausreichende Anzahl Fahrzeuge mit autonomen Fähigkeiten im Verkehr sein werden, wird es für die Effizienz des Verkehrs sehr vorteilhaft sein, autonomes Fahren auf reservierten Fahrstreifen zu konzentrieren. Durch eine Separierung kann wegen des nicht-linearen Verlaufs der Kapazität über dem Anteil autonomer Fahrzeuge der Nutzen maximiert werden. In Verbindung mit speziell gewidmeten Fahrstreifen könnte zudem die Kolonnengeschwindigkeit selbst bei größerer Verkehrsnachfrage erhöht werden, was zu weiteren deutlichen Kapazitätsgewinnen führen würde. Dies ist bei gemischtem Verkehr nicht möglich, da selbst bei wenigen von Menschen gelenkten Fahrzeugen diese die Geschwindigkeit vorgeben würden.

Im Rahmen einer ersten Auseinandersetzung mit dem weitreichenden Thema wurden bewusst in diesem Beitrag ausschließlich die verkehrlichen Wirkungen autonomer Fahrzeuge auf Streckenabschnitten von Autobahnen und - repräsentativ für den Stadtverkehr - an Knotenpunkten mit Lichtsignalanlage untersucht. Diese beiden Fahrsituationen bestimmen zu einem hohen Anteil die Qualität des Verkehrsablaufs. Daneben gibt es jedoch eine Reihe weiterer relevanter Fahrsituationen, die auf die Kapazität des Gesamtsystems einen signifikanten Einfluss haben können:

1. Außerorts sind dies an den Knotenpunkten der Fernstraßen die Einfädel-, Ausfädelund Verflechtungsmanöver. In diesem Zusammenhang sind einerseits die technischen Lösungen, die sich bereits heute mit Assistenzfunktionen wie den Einfädelungsassistenten abzeichnen, in ihrer weiteren Entwicklung insbesondere in Hinsicht auf die Möglichkeiten der maschinellen Kooperation vorauszudenken. Andererseits sind Lösungsansätze für die baulichen und regulatorischen Anpassungen der Verkehrsanlagen zu entwickeln.

So erscheint beispielsweise ein Szenario interessant, in dem der autonome Verkehr zwischen den Anschlussstellen der Autobahnen auf separaten Fahrstreifen geführt und 
im Knotenpunktbereich die Separierung aufgehoben wird. Im Knotenpunktbereich treten damit autonome und von Menschen gesteuerte Fahrzeuge auf allen Fahrstreifen auf und können jeweils alle Fahrmanöver (autonom, hoch unterstützt oder durch Menschen gesteuert) bei einer möglicherweise vorgegebenen niedrigen Geschwindigkeit durchführen.

2. Innerorts werden insbesondere noch Fragen des Einflusses der sogenannten bedingten Verträglichkeit zu klären sein. Bedingte Verträglichkeit tritt dann auf, wenn sich kreuzende Verkehrsströme an Lichtsignalanlagen gleichzeitig freigegeben werden und Vorfahrtsregeln eingehalten werden müssen. Dies ist z. B. der Fall bei rechts- oder linksabbiegenden Fahrzeugströmen, die dem parallel geführten Fußgänger- und Radverkehr Vorrang einräumen müssen. Verschiedenste Lösungsansätze könnten hierfür interessant sein und sollten einer genaueren Überprüfung unterzogen werden. So könnte man alle Fahrstreifen der autonomen Fahrzeuge gleichzeitig in einer eigenen Phase freigeben - die Fahrmanöver der feindlichen Ströme im Knotenpunktbereich würden von den autonomen Fahrzeugen selbstständig ausgehandelt werden. Alle anderen Verkehrsteilnehmer würden mit der bereits bestehenden Signalisierung behandelt werden. Eine andere Lösungsmöglichkeit wäre die Berücksichtigung der Radfahrer und Fußgänger in einer eigenen Phase mit „Rundumgrün“ bei gleichzeitiger Vermeidung von bedingt verträglichen Kfz-Strömen durch eine geeignete Phasenstruktur.

\subsubsection{Kooperation}

Für Szenarien wie das zuletzt angesprochene des sich selbst organisierenden kreuzenden Verkehrs benötigen autonome Fahrzeuge die Fähigkeit der Kommunikation untereinander und mit der Infrastruktur. Die Antizipation von Manövern von vorausfahrenden Fahrzeugen und die davon abhängenden Reaktionen in der darauffolgenden Kolonne wirken sich auf komfortable und damit akzeptable Beschleunigungswerte und ein komfortables Fahrgefühl aus. Deshalb wird den bereits heute entwickelten Technologien zur Kommunikation und Kooperation bei der Entwicklung des autonomen Fahrens eine wichtige Rolle zukommen.

\section{Literatur}

1. Brilon, W. ; Regler, M., Geistefeldt, J (2005): Zufallscharakter der Kapazität von Autobahnen und praktische Konsequenzen. Straßenverkehrstechnik, Heft 3 (Teil 1) und Heft 4 (Teil 2)

2. Friedrich, B.; Kemper, C. (2006): Akzeptanz von Harmonisierungsmaßnahmen im Zuge von Verkehrsbeeinflussungsanlagen. Schlussbericht für die Niedersächsische Landesbehörde für Straßenbau und Verkehr, November 2006, Hannover

3. FGSV - Forschungsgesellschaft für Straßen- und Verkehrswesen (2001): Handbuch für die Bemessung von Straßenverkehrsanlagen (HBS), S. 3-19 
4. FGSV - Forschungsgesellschaft für Straßen- und Verkehrswesen (2014): Handbuch für die Bemessung von Straßenverkehrsanlagen (HBS) - Entwurf der Neufassung

5. Greenshields, B.D. (1935): A study of highway capacity. Proceedings Highway Research Record, Washington Volume 14, pp. 448-477

6. Hall, F. L.; Agyemang-Duah, K. (1991): Freeway capacity drop and the definition of capacity. Transportation Research Record 1320, TRB, National Research Council, Washington D.C.

7. Janiszewski, H.; Jagow, J.; Burmann, M. (2005): Straßenverkehrsrecht. Beck Juristischer Verlag, München

8. Koshi, M.; Iwasaki, M.; Ohkura, I. (1981): Some findings and an overview on vehicular flow characteristics. Proc. $8^{\text {th }}$ Int. Symposium on Transportation and Traffic Theory, Ed. Hurdle, Hauer, Steward, Toronto

9. May, A.D.; Keller, H. (1968): Evaluation of single- and two-regime traffic flow models. Straßenbau und Straßenverkehrstechnik, 1968, Heft 86, S. 37-47

10. Ponzlet, M. (1996): Dynamik der Leistungsfähigkeiten von Autobahnen, Heft 16 der Schriftenreihe des Lehrstuhls für Verkehrswesen der Ruhr-Universität Bochum

11. Wagner, P. (2014 b): Private Mitteilung 